\title{
Vom reinen Infostand zur interaktiven Begegnungsstätte
}

\author{
In diesem Jahr feierte das neue Standkonzept von FMH und FMH Services seine Pre- \\ miere: Dieses setzt auf eine vermehrte Interaktion und Begegnung mit den Besu- \\ cherinnen und Besucher. Ebenfalls zum ersten Mal überhaupt waren die FMH, FMH \\ Services, EMH und HIN im laufenden Jahr mit einem gemeinsamen Stand präsent. \\ Die Standbetreiber ziehen eine positive Bilanz - und betonen den Synergie-Effekt, \\ welcher der Auftritt der vier Organisationen mit sich bringt.
}

Maximilano Wepfer

Stv. Leiter Abteilung Kommunikation FMH

Korrespondenz: FMH / Abteilung Kommunkation Elfenstrasse 18 CH-3000 Bern 15 Tel. 0313501111 Fax 0313591112
An der diesjährigen IFAS war er nicht zu übersehen: der gemeinsame Stand der FMH, FMH Services und HIN. Die drei Organisationen nahmen an der Fachmesse für den Gesundheitsmarkt teil, die vom 21. bis 24. Oktober in der Messe Zürich stattfand. «Dank der Lage gleich beim Eingang der Halle war der Stand für alle Besucherinnen und Besucher ein richtiger Eyecatcher», freut sich Christoph Kreyden, Standchef und Leiter des Dienstes Dienstleistungen und Mitgliedschaft.

Seit 2002 vertritt Kreyden regelmässig die FMH an Kongressen und Messen und hat die ganze Entwicklung miterlebt. Angefangen hat er mit einem portablen Stand, den er noch selber auf- und abgebaut hat, bis er nach vier Jahren einen Standbauer beizog - das Ganze war zu gross geworden. Nicht
Bestätigte Präsenz an Messen und Kongressen im Jahr 2015

\begin{tabular}{|c|c|c|}
\hline Veranstaltung & Datum & Ort \\
\hline JHaS-Kongress & 18.4 .2015 & Thun \\
\hline $\begin{array}{l}\text { SGIM-Jahresversamm- } \\
\text { lung }\end{array}$ & $20.5 .2015-22.5 .2015$ & Basel \\
\hline KHM-Kongress & 25.6.2015-26.6.2015 & Luzern \\
\hline Swiss Family Docs & 27.8.2015-28.8.2015 & Bern \\
\hline
\end{tabular}

alles gelang auf Anhieb: Die Präsentationen auf Flachbildschirmen im Jahr 2008 etwa kamen nicht so spektakulär wie erwartet bei den Besucherinnen und Besuchern an. «Der persönliche Kontakt ist und bleibt das Wichtigste», betont Kreyden. An Messen

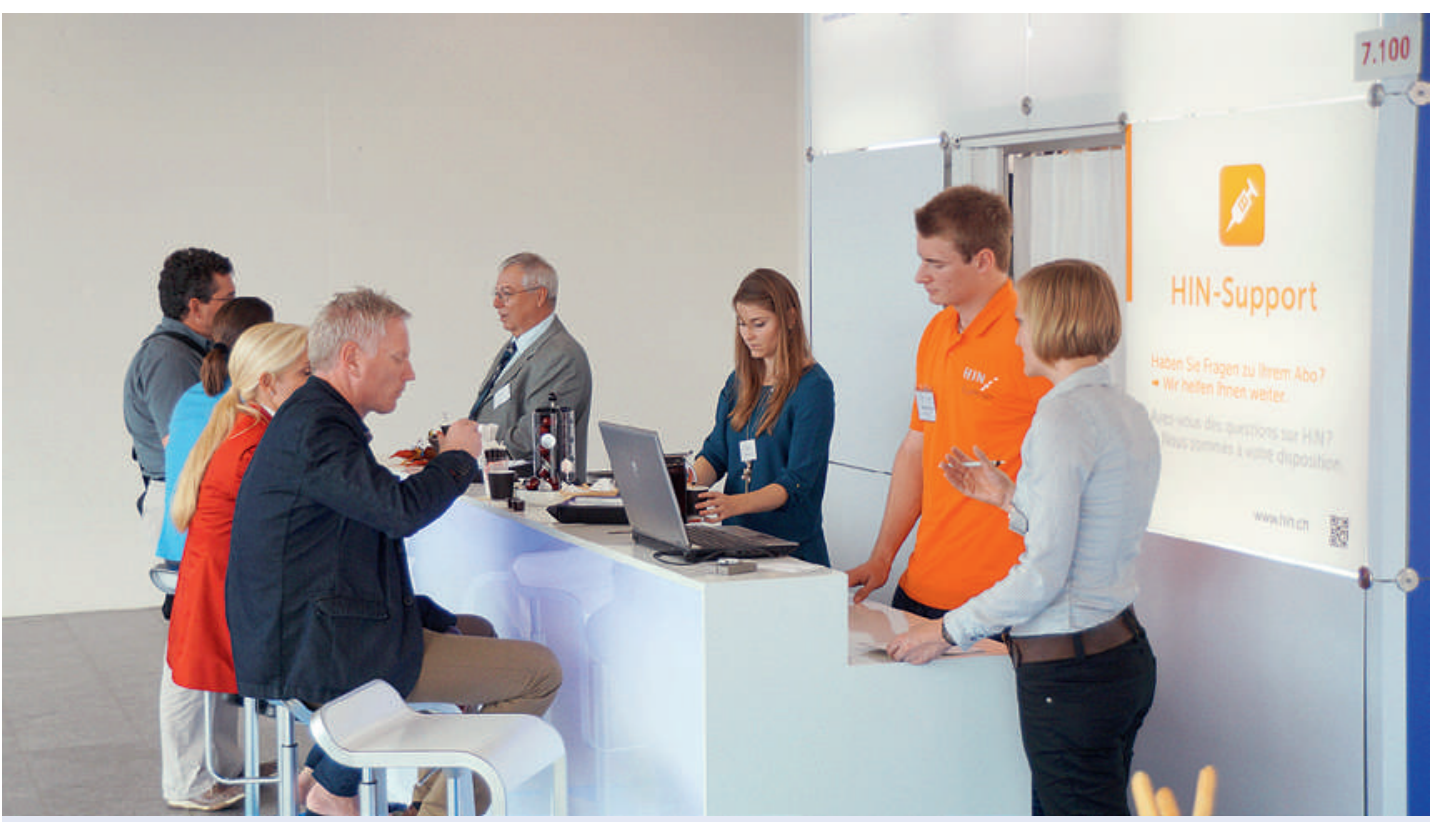

FMH-Mitglieder werden am Messestand direkt und kompetent zu ihren Anliegen beraten. 


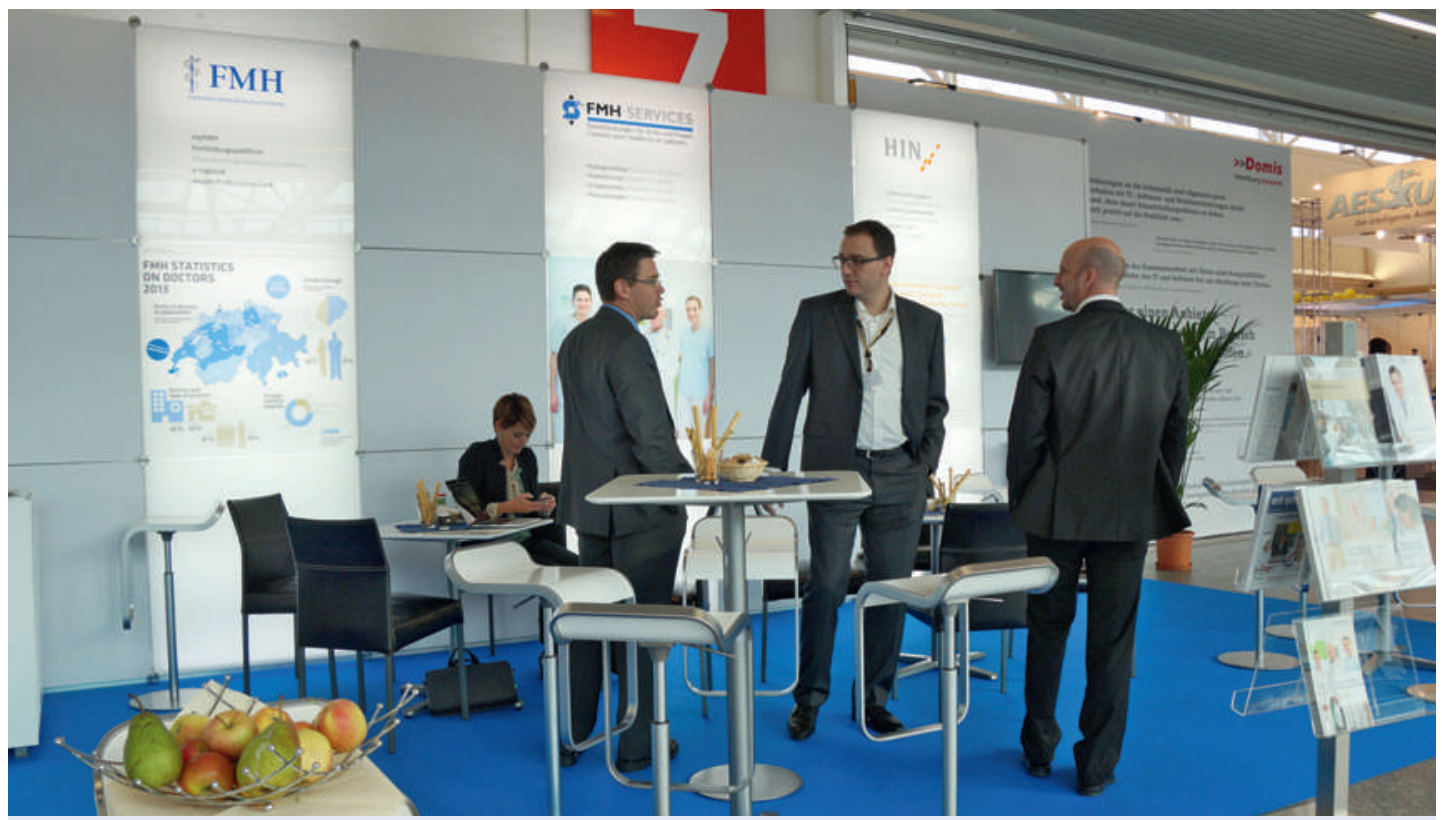

Der neu konzipierte Stand der FMH, FMH Services und HIN bietet mehr Möglichkeiten zum Dialog und zur Interaktion an.

und Kongressen können FMH-Mitglieder die vielfältigen Dienstleistungen in den Bereichen wie beispielsweise Praxisführung, Datenaustausch oder medizinische Publikationen kennenlernen sowie ihre Probleme und Anregungen von Angesicht zu Angesicht anbringen. «Wir haben dann die Möglichkeit, ihnen direkt vor Ort weiterzuhelfen oder ihr Anliegen aufzunehmen», hält er fest.
Kreyden sehr zufrieden. Durch seine modulare Struktur lässt sich der Stand ausserdem an die je nach Messe verfügbare Fläche in verschiedenen Grössen aufbauen.

Ein neuer Stand war auch notwendig, weil sich einerseits der Kreis der ausstellenden Organisationen (FMH und FMH Services) auf dieses Jahr hin um die EMH und HIN erweiterte und andererseits die Orga-

\section{Eine Integration des SIWF ist geplant, damit Besucherinnen und Besucher auch wichtige Infos zur ärztlichen Weiter- und Fortbildung erhalten.}

Mit dem Fokus auf diesem direkten Kontakt wurde beschlossen, im vergangenen Jahr das Standkonzept zu überarbeiten. Die Erfahrung hatte gezeigt, dass es aufgrund der Einrichtung des alten Standes zwischen den Standrepräsentanten und den Besuchern unbewusst eine Barriere gab, die wenig Platz zum Reden liess. «Wir wollten weg vom Infostand und hin zur Begegnungsstätte», erklärt Kreyden. Dieses Jahr erlebte das moderne Standkonzept seine Feuertaufe: Der Stand wirkt luftig und lichtdurchflutet, bietet dank mehr Tischen auch eine grössere Dialogfläche an und lädt zur Interaktion ein. «So können wir besser auf die Besucherinnen und Besucher zugehen, um sie noch persönlicher zu bedienen und kompetenter zu beraten», zeigt sich nisationen ihre Dienstleistungen besser präsentieren wollten. Kreyden schätzt die Synergien, die sich durch diese verstärkte Präsenz ergeben. «Ärztinnen und Ärzte beziehen Dienstleistungen von allen vier Organisationen», führt er aus. «Wenn sie also diese verschiedenen Dienstleistungen auf einem Platz finden, ist das eine sinnvolle Ergänzung.» Und dieser Kreis soll sich sogar vergrössern: Eine Integration des SIWF ist geplant, damit Besucherinnen und Besucher auch wichtige Infos zur ärztlichen Weiter- und Fortbildung erhalten. Für die Zukunft hofft Kreyden, dass sich das neue Standkonzept weiterhin so bewährt, wie es sich im ersten Jahr angelassen hat. Die Weiterentwicklung bleibt darüber hinaus ein Ziel für die kommenden Jahre. 